\title{
Search for Sub-eV Mass Solar Axions by the CERN Axion Solar Telescope with ${ }^{3} \mathrm{He}$ Buffer Gas
}

\author{
M. Arik, ${ }^{1, \dagger}$ S. Aune, ${ }^{2}$ K. Barth, ${ }^{3}$ A. Belov,${ }^{4}$ S. Borghi, ${ }^{3,}$ H. Bräuninger ${ }^{5}$ G. Cantatore, ${ }^{6}$ J. M. Carmona, ${ }^{7}$ S. A. Cetin, ${ }^{1}$
} J. I. Collar, ${ }^{8}$ T. Dafni, ${ }^{7}$ M. Davenport,${ }^{3}$ C. Eleftheriadis, ${ }^{9}$ N. Elias, ${ }^{3}$ C. Ezer,,${ }^{1 \dagger}$ G. Fanourakis, ${ }^{10}$ E. Ferrer-Ribas, ${ }^{2}$ P. Friedrich, ${ }^{5}$ J. Galán, ${ }^{7,}$ J. A. García, ${ }^{7}$ A. Gardikiotis, ${ }^{11}$ E. N. Gazis, ${ }^{12}$ T. Geralis, ${ }^{10}$ I. Giomataris, ${ }^{2}$ S. Gninenko, ${ }^{4}$ H. Gómez, ${ }^{7}$ E. Gruber, ${ }^{13}$ T. Guthörl, ${ }^{13}$ R. Hartmann, ${ }^{14, \|}$ F. Haug, ${ }^{3}$ M. D. Hasinoff, ${ }^{15}$ D. H. H. Hoffmann, ${ }^{16}$ F. J. Iguaz,,${ }^{7,}$ I. G. Irastorza, ${ }^{7}$ J. Jacoby, ${ }^{17}$ K. Jakovčić, ${ }^{18}$ M. Karuza, ${ }^{6}$ K. Königsmann, ${ }^{13}$ R. Kotthaus, ${ }^{19}$ M. Krčmar, ${ }^{18}$ M. Kuster, ${ }^{5,16, \pi}$ B. Lakić, ${ }^{18, * *}$ J. M. Laurent, ${ }^{3}$ A. Liolios, ${ }^{9}$ A. Ljubičić, ${ }^{18}$ V. Lozza, ${ }^{6}$ G. Lutz, ${ }^{14, \|}$ G. Luzón, ${ }^{7}$ J. Morales, ${ }^{7, *}$ T. Niinikoski, ${ }^{3, \dagger \dagger}$ A. Nordt, ${ }^{5,16,+1}$ T. Papaevangelou, ${ }^{2}$ M. J. Pivovaroff, ${ }^{20}$ G. Raffelt, ${ }^{19}$ T. Rashba,${ }^{21}$ H. Riege, ${ }^{16}$ A. Rodríguez, ${ }^{7}$ M. Rosu, ${ }^{16}$ J. Ruz, ${ }^{7,3}$ I. Savvidis, ${ }^{9}$ P. S. Silva, ${ }^{3}$ S. K. Solanki, ${ }^{21}$ L. Stewart, ${ }^{3}$ A. Tomás, ${ }^{7}$ M. Tsagri, ${ }^{11,+*}$ K. van Bibber, ${ }^{20,8 \S}$ T. Vafeiadis, ${ }^{3,9,11}$ J. A. Villar, ${ }^{7}$ J. K. Vogel, ${ }^{13,20, \| l \mid}$ S. C. Yildiz, ${ }^{1, \dagger}$ and K. Zioutas ${ }^{3,11}$

(CAST Collaboration)

\author{
${ }^{1}$ Dogus University, Istanbul, Turkey \\ ${ }^{2} I R F U$, Centre d'Études Nucléaires de Saclay (CEA-Saclay), Gif-sur-Yvette, France \\ ${ }^{3}$ European Organization for Nuclear Research (CERN), Genève, Switzerland \\ ${ }^{4}$ Institute for Nuclear Research (INR), Russian Academy of Sciences, Moscow, Russia \\ ${ }^{5}$ Max-Planck-Institut für Extraterrestrische Physik, Garching, Germany \\ ${ }^{6}$ Instituto Nazionale di Fisica Nucleare (INFN), Sezione di Trieste and Università di Trieste, Trieste, Italy \\ ${ }^{7}$ Laboratorio de Física Nuclear y Astropartículas, Universidad de Zaragoza, Zaragoza, Spain \\ ${ }^{8}$ Enrico Fermi Institute and KICP, University of Chicago, Chicago, Illinois, USA \\ ${ }^{9}$ Aristotle University of Thessaloniki, Thessaloniki, Greece \\ ${ }^{10}$ National Center for Scientific Research "Demokritos," Athens, Greece \\ ${ }^{11}$ Physics Department, University of Patras, Patras, Greece \\ ${ }^{12}$ National Technical University of Athens, Athens, Greece \\ ${ }^{13}$ Albert-Ludwigs-Universität Freiburg, Freiburg, Germany \\ ${ }^{14}$ MPI Halbleiterlabor, München, Germany \\ ${ }^{15}$ Department of Physics and Astronomy, University of British Columbia, Vancouver, Canada \\ ${ }^{16}$ Technische Universität Darmstadt, IKP, Darmstadt, Germany \\ ${ }^{17}$ Johann Wolfgang Goethe-Universität, Institut für Angewandte Physik, Frankfurt am Main, Germany \\ ${ }^{18}$ Rudjer Bošković Institute, Zagreb, Croatia \\ ${ }^{19}$ Max-Planck-Institut für Physik (Werner-Heisenberg-Institut), München, Germany \\ ${ }^{20}$ Lawrence Livermore National Laboratory, Livermore, California, USA \\ ${ }^{21}$ Max-Planck-Institut für Sonnensystemforschung, Katlenburg-Lindau, Germany \\ (Received 21 June 2011; published 23 December 2011)
}

The CERN Axion Solar Telescope (CAST) has extended its search for solar axions by using ${ }^{3} \mathrm{He}$ as a buffer gas. At $T=1.8 \mathrm{~K}$ this allows for larger pressure settings and hence sensitivity to higher axion masses than our previous measurements with ${ }^{4} \mathrm{He}$. With about $1 \mathrm{~h}$ of data taking at each of 252 different pressure settings we have scanned the axion mass range $0.39 \mathrm{eV} \lesssim m_{a} \lesssim 0.64 \mathrm{eV}$. From the absence of excess $\mathrm{x}$ rays when the magnet was pointing to the Sun we set a typical upper limit on the axion-photon coupling of $g_{a \gamma} \leqslant 2.3 \times 10^{-10} \mathrm{GeV}^{-1}$ at $95 \%$ C.L., the exact value depending on the pressure setting. Kim-Shifman-Vainshtein-Zakharov axions are excluded at the upper end of our mass range, the first time ever for any solar axion search. In the future we will extend our search to $m_{a} \lesssim 1.15 \mathrm{eV}$, comfortably overlapping with cosmological hot dark matter bounds.

DOI: 10.1103/PhysRevLett.107.261302

PACS numbers: 95.35.+d, 14.80.Va, 96.60.Vg

Published by the American Physical Society under the terms of the Creative Commons Attribution 3.0 License. Further distribution of this work must maintain attribution to the author(s) and the published article's title, journal citation, and DOI.
Introduction.-The Peccei-Quinn mechanism is the most compelling explanation for why in QCD the $\Theta$ term does not cause measurable $C P$-violating effects such as a large neutron electric dipole moment [1-3]. A testable consequence is the existence of axions, low-mass pseudoscalar bosons that are closely related to neutral pions. The axion mass is given by $m_{a} f_{a} \sim m_{\pi} f_{\pi}$ and the two-photon 
interaction strength scales with $f_{\pi} / f_{a}$ where $f_{\pi} \sim$ $92 \mathrm{MeV}$ is the pion decay constant and $f_{a}$ a large energy scale related to the breaking of a new U(1) symmetry of which the axion is the Nambu-Goldstone boson.

Axions would have been produced in the early Universe by the vacuum realignment mechanism and radiation from cosmic strings, leading to a cold dark matter component, as well as from thermal interactions, leading to a hot dark matter component [4,5]. Precision cosmology requires $m_{a} \lesssim 0.9 \mathrm{eV}$ for the latter [6,7], with the usual caveats concerning systematic uncertainties. The cold component increases with decreasing $m_{a}$ and provides all dark matter for $m_{a} \sim 10 \mu \mathrm{eV}\left(f_{a} \sim 10^{12} \mathrm{GeV}\right)$, with large uncertainties depending on the early Universe scenario. The ongoing ADMX dark matter search [8], based on Sikivie's idea of axion-photon conversion in a macroscopic $B$ field [9], provides one of the few realistic opportunities to find "invisible axions" [10].

Axions would also emerge from the hot interiors of stars, the Sun being the most powerful "local" source [11]. To search for these axions, one can use magnetically induced a $\gamma$ conversion in a dipole magnet pointing toward the Sun ("axion helioscope" technique [9]). This is analogous to neutrino flavor oscillations, $a \gamma$ mixing being caused by the $B$ field [12]. The axion-photon interaction is given by Lagrangian $\mathcal{L}_{a \gamma}=g_{a \gamma} \mathbf{E} \cdot \mathbf{B} a$ with $g_{a \gamma}=\left(\alpha / 2 \pi f_{a}\right) \times$ $[E / N-2(4+z) / 3(1+z)]$. Here $z=m_{u} / m_{d}$ with the canonical value 0.56 , although the range $0.35-0.60$ is possible [3]. $E / N$ is a model-dependent ratio of small integers [13] and $E / N=0$ [Kim-Shifman-Vainshtein-Zakharov (KSVZ) model $[14,15]]$ is our benchmark case (Fig. 1).

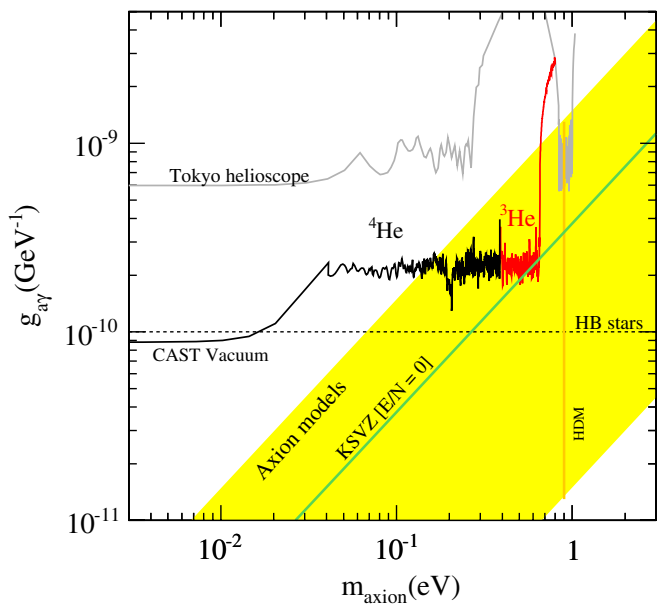

FIG. 1 (color online). Exclusion regions in the $m_{a}-g_{a \gamma}$ plane achieved by CAST in the vacuum [20,21], ${ }^{4} \mathrm{He}$ [22], and ${ }^{3} \mathrm{He}$ phase. We also show constraints from the Tokyo helioscope [17-19], horizontal branch (HB) stars [11], and the hot dark matter (HDM) bound [6]. The yellow band labeled "Axion models" represents typical theoretical models with $\mid E / N-$ $1.95 \mid=0.07-7$. The green solid line inside the band corresponds to $E / N=0$ (KSVZ model).
After a pioneering axion helioscope in Brookhaven [16], a fully steerable instrument was built in Tokyo [17-19]. The largest helioscope yet is the CERN Axion Solar Telescope (CAST), using a refurbished LHC test magnet ( $L=9.26 \mathrm{~m}, B \sim 9.0 \mathrm{~T}$ ) mounted to follow the Sun for about $1.5 \mathrm{~h}$ both at dawn and dusk [20-24]. CAST began operation in 2003 and after two years of data taking with vacuum inside the magnet bores achieved a limit of $g_{a \gamma}<0.88 \times 10^{-10} \mathrm{GeV}^{-1}$ at $95 \%$ C.L. for $m_{a} \lesssim 0.02 \mathrm{eV}$ $[20,21]$. While these results are excellent to constrain very light axionlike particles [25], realistic QCD axions are not covered because the $g_{a \gamma}$ bounds quickly degrade for $m_{a} \gtrsim 0.02 \mathrm{eV}$ (Fig. 1).

Sensitivity to higher axion masses improves if the conversion volume contains a buffer gas such as helium [26]. Then the $a \gamma$ conversion probability is

$$
P_{a \rightarrow \gamma}=\left(\frac{B g_{a \gamma}}{2}\right)^{2} \frac{1+e^{-\Gamma L}-2 e^{-\Gamma L / 2} \cos (q L)}{q^{2}+\Gamma^{2} / 4},
$$

where $\Gamma$ is the inverse photon absorption length in the buffer gas, while the momentum difference between the $a$ and $\gamma$ propagation eigenstates is given by $q^{2}=\left[\left(m_{a}^{2}-\right.\right.$ $\left.\left.m_{\gamma}^{2}\right) / 2 E\right]^{2}+\left(g_{a \gamma} B\right)^{2}$. For $m_{a}^{2}=m_{\gamma}^{2}$, axions and photons are maximally mixed and reach $P_{a \rightarrow \gamma}=\left(g_{a \gamma} B L / 2\right)^{2}=$ $1.7 \times 10^{-17}$ for $L=9.26 \mathrm{~m}, B=9.0 \mathrm{~T}$, and $g_{a \gamma}=$ $10^{-10} \mathrm{GeV}^{-1}$. For $m_{a} \neq m_{\gamma}$, the conversion probability rapidly decreases due to the axion-photon momentum mismatch.

The maximum $P_{a \rightarrow \gamma}$ can be restored by matching $m_{a}$ with a photon refractive mass $m_{\gamma}$ [26]. This method was first applied by the Brookhaven helioscope using ${ }^{4} \mathrm{He}$ as a buffer gas [16] and later allowed CAST to reach realistic axion models for $m_{a} \lesssim 0.4 \mathrm{eV}$ (Fig. 1) [22]. However, $T=1.8 \mathrm{~K}$ of the superconducting magnet restricts, due to condensation, the maximum ${ }^{4} \mathrm{He}$ pressure to $\sim 14 \mathrm{mbar}$ thus allowing us to scan axion masses $m_{a} \lesssim 0.4 \mathrm{eV}$. To close the gap to the hot-dark matter bound, we have used ${ }^{3} \mathrm{He}$ as buffer gas to allow CAST to search up to $m_{a} \lesssim$ $1.15 \mathrm{eV}$. The first results from this novel technique for the axion mass range $0.39 \lessgtr m_{a} \lesssim 0.64 \mathrm{eV}$ are reported here.

Upgrades.-After completing the data taking with ${ }^{4} \mathrm{He}$ as a buffer gas, the CAST experiment performed several upgrades in order to prepare for data taking with ${ }^{3} \mathrm{He}$. The most important upgrade was the design and installation of a sophisticated ${ }^{3} \mathrm{He}$ gas system.

To scan over a range of axion masses, CAST needs to control precisely the helium gas density in the cold bores. This is achieved by filling the cold bores with a precisely metered amount of gas in incremental steps. The step size of the gas density is equivalent to a pressure change of between 0.083 and $0.140 \mathrm{mbar}$ (calculated for gas at nominal temperature of $1.8 \mathrm{~K}$ ). To scan the whole available mass range efficiently, data taking runs cover two density settings per solar tracking. During the measurement, it is 
desired to have the gas density in the cold bores to be as homogenous and as stable as possible. The density homogeneity is ensured by the excellent thermal coupling with the superfluid helium bath surrounding the cold bores. To achieve the proper densities in the cold bores of CAST, and to be able to reproducibly refill the bores (allowing us to search the same axion mass), requires that the gas system is capable of adjusting to fluctuations of external conditions (e.g., variations of the room and magnet temperatures). The density stability due to uncorrelated temperature fluctuations is met by minimizing the volume of external pipework connected to the cold bore. The density fluctuations are well within the density stability limit of $0.001 \mathrm{~kg} / \mathrm{m}^{3}$ (for example, the allowed magnet temperature fluctuations are about $350 \mathrm{mK}$ while typical fluctuation during magnet vertical movement is $35 \mathrm{mK}$ ).

The ${ }^{3} \mathrm{He}$ system can be described as a hermetically closed gas circuit which is divided into functional sections with specific purposes: Storage, trap purge system, metering and ramping of gas density, expansion volume, recovery, and circulation.

All the necessary helium for CAST physics runs is transferred to the storage volume that has been specifically engineered to keep the gas pressure below atmospheric. Before entering the metering volumes, the gas passes through two charcoal traps. The first one at ambient temperature traps oil and water vapor while the second at liquid nitrogen temperature removes residual gases.

The metering precision of the gas density is obtained by the accurate temperature control of the metering volumes, and by use of metrology-grade pressure-measuring instruments to determine the amount of gas introduced into the cold bores. This amount of gas is calculated by accurately measuring the pressure decrease in the metering volumes. The reproducibility for the amount of gas sent from the metering volume into the magnet is $61 \mathrm{ppm}$.

The gas is confined in the cold bore region of the magnet with thin x-ray windows installed on both ends. The windows are made of $15 \mu \mathrm{m}$-thick polypropylene stretched over a mostly open strongback structure to provide high $\mathrm{x}$-ray transmission, resistance to a sudden rise in pressure and minimal helium leakage. Heaters on the window flanges allow for periodic bakeout of gases adsorbed on the polypropylene.

In case of quench, a sudden loss of superconductivity in the magnet, the temperature of the magnet increases rapidly. If the cold volume remains closed, the gas pressure abruptly increases and endangers the integrity of the $\mathrm{x}$-ray windows. The windows can safely withstand pressures up to $1.2 \mathrm{bar}$, and to prevent rupture during a quench, the system must safely evacuate the ${ }^{3} \mathrm{He}$ from the cold bores to the expansion volume. Thus, the expansion volume, initially under vacuum, acts as a buffer reservoir for the gas that is intentionally expelled from the cold bores. The CAST ${ }^{3} \mathrm{He}$ system will be described in detail in a future publication.
It is a demanding task to compute the amount of gas needed to achieve the desired gas density. In fact, such calculations can only reliably be performed through computational fluid dynamic (CFD) simulations that account for the as-built system, as well as different physical phenomena such as hydrostatic effects, convection, and buoyancy. For a typical run, e.g., $m_{\gamma}=0.64 \mathrm{eV}$, the intrinsic mass-acceptance width coming from the coherence condition [22] increases due to the mentioned phenomena from 0.8 to $1.6 \mathrm{meV}$ while the height decreases accordingly. The CFD simulations will be described in detail in a future publication.

During preparations for the ${ }^{3} \mathrm{He}$ data taking, the CAST $\mathrm{x}$-ray detectors were upgraded as well. The time projection chamber (TPC) with a multiwire proportional readout [27] that had covered both bores of the sunset end of the magnet was replaced by two Micromegas detectors of similar dimensions of the one previously installed at the sunrise side [28] but with readouts fabricated with novel bulk and microbulk techniques [29-31]. On the sunrise end a new shielded bulk (and later on microbulk) Micromegas replaced the unshielded one of our previous run [28]. These novel techniques provide several improvements in terms of stability and homogeneity of response, energy resolution, simplicity of construction [29-31] and, for the case of microbulk readouts, material radiopurity [32]. This is the first time these kinds of readouts are used in a physics run of a low background experiment. These new Micromegas detectors have obtained background levels down to $\sim 5 \times 10^{-6}$ counts $\mathrm{keV}^{-1} \mathrm{~cm}^{-2} \mathrm{~s}^{-1}$ in the energy range of interest, 1 order of magnitude better than their predecessors [22]. This improvement is due to new shielding in the case of the sunrise detector, and to better rejection capabilities of the Micromegas readout with respect to the MWPC one, for the sunset setup. The remaining background is attributed to unshielded external gammas (mostly due to the solid angle of incomplete shielding on the side where the detector is connected to the magnet bore). The $\mathrm{x}$-ray mirror telescope with a pn-CCD chip [33] covering the other bore of the sunrise side remained unchanged.

Data analysis and results. - Data presented in this Letter correspond to the first 252 density steps of the ${ }^{3} \mathrm{He}$ phase, which encompass an equivalent axion mass range between 0.39 and $0.64 \mathrm{eV}$. The total available exposure time in axion-sensitive conditions is about $200 \mathrm{~h}$ per detector, shared approximately equally among each of the four CAST detectors, as well as among the stated range of axion masses.

Data analysis is performed in a manner similar to our previous results obtained with ${ }^{4} \mathrm{He}$ gas. This time, however, we use an unbinned likelihood function that can be expressed as

$$
\log L \propto-R_{T}+\sum_{i}^{N} \log R\left(t_{i}, E_{i}, d_{i}\right),
$$


where the sum runs over each of the $N$ detected counts and $R\left(t_{i}, E_{i}, d_{i}\right)$ is the event rate expected at the time $t_{i}$, energy $E_{i}$ and detector $d_{i}$ of the event $i . R_{T}$ is the integrated expected number of counts over all exposure time, energy and detectors

$$
R(t, E, d)=B_{d}+S(t, E, d),
$$

where $B_{d}$ is the background rate of detector $d . S(t, E, d)$ is the expected rate from axions in detector $d$ which depends on the axion properties $g_{a \gamma}$ and $m_{a}$

$$
S(t, E, d)=\frac{d \Phi_{a}}{d E} P_{a \rightarrow \gamma} \epsilon_{d}
$$

where $P_{a \rightarrow \gamma}$ is the axion-photon conversion probability in the CAST magnet (1), $\epsilon_{d}$ the detector efficiency, and

$$
\frac{d \Phi_{a}}{d E}=6.02 \times 10^{10} g_{10}^{2} \frac{E^{2.481}}{e^{E / 1.205}} \mathrm{~cm}^{-2} \mathrm{~s}^{-1} \mathrm{keV}^{-1}
$$

is the solar axion spectrum, with $g_{10}=g_{a \gamma} /$ $\left(10^{-10} \mathrm{GeV}^{-1}\right)$ and energies in $\mathrm{keV}$.

As explained in [22], the $m_{a}$ dependency of the above expression is encoded in the probability $P_{a \rightarrow \gamma}$, which is coherently enhanced for values of $m_{a}$ matching the photon mass $m_{\gamma}$ induced by the buffer gas density, while it is negligible for values away from $m_{\gamma}$. Therefore, only the counts observed with the gas density matching a given axion mass $m_{a}$ will contribute to the $\log L$ (and the exclusion plot) for that mass $m_{a}$.

The use of the unbinned likelihood (2), instead of the binned one used in our previous result [22] is motivated by the overall reduction of background rates achieved by CAST detectors with respect to the ones of the ${ }^{4} \mathrm{He}$ phase, as well as due to the reduced ${ }^{3} \mathrm{He}$ density setting exposure time (one-half that for ${ }^{4} \mathrm{He}$ ) due to time constraints of the overall data taking campaign. Indeed, the effective number of background counts in this analysis is about 1 count per density step for the Micromegas detectors, and about 0.2 in the fiducial spot of the CCD/Telescope system. Because of that, the result obtained is almost statistics limited, and further background reduction would give only slightly better sensitivity unless longer exposure times are available.

The remaining process is similar to the one followed in our previous results [22]: a best fit value $g_{\min }^{4}$ is obtained after maximization of $L$ (for a fixed value of $m_{a}$ ). The obtained value is compatible with the absence of positive signal, and therefore an upper limit $g_{95}^{4}$ is obtained by integration of the Bayesian probability from zero up to $95 \%$ of its area in $g^{4}$. This value is computed for many values of the axion mass $m_{a}$ in order to configure the full exclusion plot shown in Fig. 1. A close up of the same exclusion plot is shown in Fig. 2, focused specifically in the axion mass range which has been explored in the data presented here.

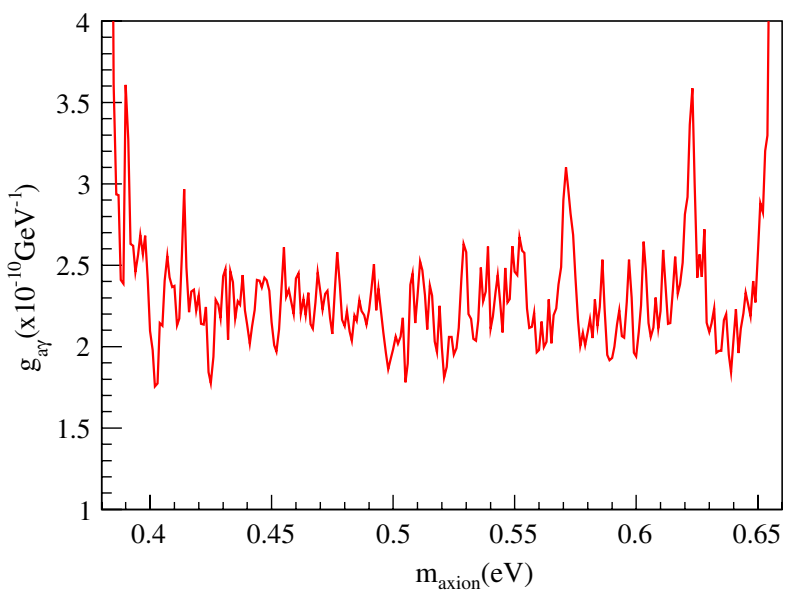

FIG. 2 (color online). Expanded view of the limit achieved in the ${ }^{3} \mathrm{He}$ CAST phase for axion mass range between 0.39 and $0.64 \mathrm{eV}$.

As can be seen in Fig. 1, CAST extends its previous exclusion plot towards higher axion masses, excluding the interval $0.39-0.64 \mathrm{eV}$ down to an average value of the axion-photon coupling of $2.27 \times 10^{-10} \mathrm{GeV}^{-1}$. The actual limit contour has high-frequency structure that is a result of statistical fluctuations that occur when a limit is computed for a specific mass using only a few hours of data.

Conclusions. - CAST has taken a great leap forward by using ${ }^{3} \mathrm{He}$ as buffer gas to cover $m_{a}$ in the gap between our ${ }^{4} \mathrm{He}$ results and the hot dark matter bound. It is the first axion helioscope ever that has crossed the "axion line" for the benchmark KSVZ case. After covering $0.39 \mathrm{eV} \lesssim$ $m_{a} \lesssim 0.64 \mathrm{eV}$ we will eventually reach $1.15 \mathrm{eV}$ with the ${ }^{3} \mathrm{He}$ setup. If axions are not detected by CAST, the next challenge is to move down in the $m_{a}-g_{a \gamma}$ plot below the "axion band" of theoretical models. Such a goal cannot be achieved with the existing CAST apparatus and will require significant improvements of detector and magnet properties $[34,35]$ or a completely new approach.

We thank CERN for hosting the experiment and for the technical support to operate the magnet and cryogenics. We thank the CERN CFD team for their essential contribution to the CFD work. We acknowledge support from NSERC (Canada), MSES (Croatia) under Grant No. 0980982887-2872, CEA (France), BMBF (Germany) under Grants No. 05 CC2EEA/9 and No. 05 CC1RD1/0 and DFG (Germany) under Grants No. HO 1400/7-1 and No. EXC-153, the Virtuelles Institut für Dunkle Materie und Neutrinos-VIDMAN (Germany), GSRT (Greece), RFFR (Russia), the Spanish Ministry of Science and Innovation (MICINN) under Grants No. FPA2007-62833 and No. FPA2008-03456, Turkish Atomic Energy Authority (TAEK), NSF (USA) under No. 0239812, U.S. Department of Energy, NASA under Grant No. NAG510842. Part of this work was performed under the auspices of the U.S. Department of Energy by Lawrence Livermore 
National Laboratory under Contract No. DE-AC5207NA27344. We acknowledge the helpful discussions within the network on direct dark matter detection of the ILIAS integrating activity (Contract No. RII3-CT-2003506222).

*Deceased.

†Present address: Bogazici University, Istanbul, Turkey.

${ }^{\ddagger}$ Present address: Department of Physics and Astronomy, University of Glasgow, Glasgow, U.K.

${ }^{\S}$ Present address: IRFU, Centre d'Études Nucléaires de Saclay (CEA-Saclay), Gif-sur-Yvette, France.

"Present address: PNSensor GmbH, München, Germany.

IIPresent address: European XFEL GmbH, Notkestrasse 85, 22607 Hamburg, Germany.

$* *$ Corresponding author.

Biljana.Lakic@irb.hr

${ }^{\dagger}$ Present address: Excellence Cluster Universe, Technische Universität München, Garching, Germany.

$\$$ Present address: European Organization for Nuclear Research (CERN), Genève, Switzerland.

$\S \S$ Present address: Naval Postgraduate School, Monterey, CA, USA.

${ }^{\|I\|}$ Present address: Lawrence Livermore National Laboratory, Livermore, CA, USA.

[1] R. D. Peccei, Lect. Notes Phys. 741, 3 (2008).

[2] J. E. Kim and G. Carosi, Rev. Mod. Phys. 82, 557 (2010).

[3] K. Nakamura et al. (Particle Data Group), J. Phys. G 37, 075021 (2010).

[4] P. Sikivie, Lect. Notes Phys. 741, 19 (2008).

[5] O. Wantz and E. P. S. Shellard, Phys. Rev. D 82, 123508 (2010).

[6] S. Hannestad, A. Mirizzi, G. G. Raffelt, and Y. Y. Y. Wong, J. Cosmol. Astropart. Phys. 08 (2010) 001.

[7] D. Cadamuro, S. Hannestad, G. Raffelt, and J. Redondo, J. Cosmol. Astropart. Phys. 02 (2011) 003.

[8] S. J. Asztalos et al. (ADMX Collaboration), Phys. Rev. Lett. 104, 041301 (2010).
[9] P. Sikivie, Phys. Rev. Lett. 51, 1415 (1983); 52, 695(E) (1984).

[10] S. J. Asztalos et al., Annu. Rev. Nucl. Part. Sci. 56, 293 (2006).

[11] G. G. Raffelt, Lect. Notes Phys. 741, 51 (2008).

[12] G. Raffelt and L. Stodolsky, Phys. Rev. D 37, 1237 (1988).

[13] S. L. Cheng, C. Q. Geng, and W. T. Ni, Phys. Rev. D 52, 3132 (1995).

[14] J. E. Kim, Phys. Rev. Lett. 43, 103 (1979).

[15] M. A. Shifman, A. I. Vainshtein, and V. I. Zakharov, Nucl. Phys. B166, 493 (1980).

[16] D. M. Lazarus et al., Phys. Rev. Lett. 69, 2333 (1992).

[17] S. Moriyama et al., Phys. Lett. B 434, 147 (1998).

[18] Y. Inoue et al., Phys. Lett. B 536, 18 (2002).

[19] Y. Inoue et al., Phys. Lett. B 668, 93 (2008).

[20] K. Zioutas et al. (CAST Collaboration), Phys. Rev. Lett. 94, 121301 (2005).

[21] S. Andriamonje et al. (CAST Collaboration), J. Cosmol. Astropart. Phys. 04 (2007) 010.

[22] E. Arik et al. (CAST Collaboration), J. Cosmol. Astropart. Phys. 02 (2009) 008.

[23] S. Andriamonje et al. (CAST Collaboration), J. Cosmol. Astropart. Phys. 12 (2009) 002.

[24] S. Andriamonje et al. (CAST Collaboration), J. Cosmol. Astropart. Phys. 03 (2010) 032.

[25] J. Jaeckel and A. Ringwald, Annu. Rev. Nucl. Part. Sci. 60, 405 (2010).

[26] K. van Bibber, P. M. McIntyre, D.E. Morris, and G. G. Raffelt, Phys. Rev. D 39, 2089 (1989).

[27] D. Autiero et al., New J. Phys. 9, 171 (2007).

[28] P. Abbon et al., New J. Phys. 9, 170 (2007).

[29] S. Andriamonje et al., JINST 5, P02001 (2010).

[30] J. Galan et al., JINST 5, P01009 (2010).

[31] S. Aune et al. (CAST Collaboration), Nucl. Instrum. Methods Phys. Res., Sect. A 604, 15 (2009).

[32] S. Cebrian et al., Astropart. Phys. 34, 354 (2011).

[33] M. Kuster et al., New J. Phys. 9, 169 (2007).

[34] I. G. Irastorza et al., J. Cosmol. Astropart. Phys. 06 (2011) 013.

[35] O. K. Baker et al., arXiv:1110.2180. 